\title{
ASCA and ANCA among Bedouin Arabs with inflammatory bowel disease, the frequency and phenotype correlation
}

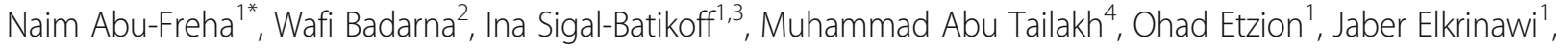
Arik Segal ${ }^{1}$, Alex Mushkalo ${ }^{1}$ and Alex Fich ${ }^{1}$

\begin{abstract}
Background: Serological markers used for diagnostic purposes and disease stratification in inflammatory bowel disease.

We aimed to investigate the frequency of ASCA and ANCA among Arab Bedouin IBD patients and its relationship to disease phenotype and course.

Methods: From cohort of 68, 25 Crohn's disease (CD) and 25 Ulcerative colitis (UC) patients were recruited (72\%). ASCA IgG was determined by ELISA assay. Immunofluorescence analysis of ANCA was performed.

Results: The IgG ASCA was detected in 13 (52\%) of the CD patients and in three (12\%) UC patients. The prevalence of ANCA among UC patients was positive with 76\%, sub-grouped, atypical ANCA in 9 patients (36\%), pANCA in six patients (24\%) and cANCA in 4 patients (16\%).

The detection of ASCA among CD patients was found not to be a reliable predictor of young age at diagnosis, gender, ileal involvement, anti-TNF treatment or surgery.

UC patients with positive ANCA were younger, mean age 40.2 \pm 11.9 compared with $57.3 \pm 21.2(p=0.03)$, and diagnosed at a younger age, $29.2 \pm 11.8$ compared with $43.5 \pm 15.3(p=0.05)$.

Conclusion: The frequency of ASCA among Bedouin CD patients and ANCA among UC patients was high, however ASCA was not found to have a predictive value for disease phenotype or course. Positive ANCA in UC patients was predictive for younger age and age at diagnosis.
\end{abstract}

Keywords: Inflammatory bowel disease, ASCA, ANCA, Southern Israel, Arab Bedouin

\section{Background}

Inflammatory bowel disease (IBD) is a chronic inflammatory disorder of the gastrointestinal tract with two major forms, Crohn's disease (CD) and ulcerative colitis (UC).

The Bedouin Arab populations are Muslims, who live in three areas in Israel, southern Israel (Negev desert), middle Israel and the Galil. In Southern Israel there are about 240.000 Arab Bedouins [1].

\footnotetext{
* Correspondence: abufreha@yahoo.de; naimaf@clalit.org.il

${ }^{1}$ The Institute of Gastroenterology and Hepatology, Soroka University Medical Center and the Faculty of Health Sciences, Ben-Gurion University of the Negev, P.O. Box 151, 84101 Beer-Sheva, Israel

Full list of author information is available at the end of the article
}

Our data from a previous report showed that there is an increase of the prevalence of (IBD) among the Bedouin population which could be attributed to the urbanization and modernization process with far-reaching lifestyle changes [2].

Diagnosis of IBD is based on a combination of clinical, radiological, endoscopic and histological criteria. Anti-Saccharomyces cerevisiae antibodies (ASCA) are antibodies directed against oligomannosidic epitopes of Saccharomyces cerevisiae, a strain of brewer's yeast [3].

Anti-neutrophil cytoplasmic antibodies (ANCA) are autoantibodies, the major ANCA antigen targets in inflammatory vasculitides is the proteinase-3, which are cytoplasmic granular with accentuation between nuclear lobes (cANCA), wherever in IBD the myeloperoxidase are the

(c) The Author(s). 2018 Open Access This article is distributed under the terms of the Creative Commons Attribution 4.0 International License (http://creativecommons.org/licenses/by/4.0/), which permits unrestricted use, distribution, and reproduction in any medium, provided you give appropriate credit to the original author(s) and the source, provide a link to the Creative Commons license, and indicate if changes were made. The Creative Commons Public Domain Dedication waiver (http://creativecommons.org/publicdomain/zero/1.0/) applies to the data made available in this article, unless otherwise stated. 
target, which are fine homogenous, diffuse rim-like staining of perinuclear cytoplasm (pANCA) [4-6].The atypical pattern of ANCA is described as fine rime-like staining with intranuclear foci or broad nonhomogenous rim-like staining of the nuclear periphery as a mixture of c-ANCA and p-ANCA $[7,8]$.

In Western countries, increased levels of ASCA were found in $50-80 \%$ of patients with CD, in $20-25 \%$ of unaffected first-degree $\mathrm{CD}$ relatives, and only in a minority of UC patients (5-15\%) and healthy subjects (0-5\%) [9-13].

Positive p-ANCA are more common among UC patients, with the frequency of $40-80 \%$ of the UC patients [14].

The role of ANCA and ASCA in IBD though still controversial could have a diagnostic role, particularly in unclear cases, a previous report found a correlation with disease behavior. ASCA and ANCA may predict the development of IBD years before it is clinically diagnosed at the early age of disease onset, fibrostenosis, internal fistulas, perianal disease and ileal involvement, ASCA positive $C D$ patients had an increased risk for early surgery $[13,15-18]$.

\section{Objective}

Our aim of the present study was to investigate the frequency of ASCA and ANCA as serologic markers of IBD among the Bedouin Arab IBD patients. A second aim was to assess the association between the presence of ASCA or ANCA and clinical features of the disease.

\section{Methods}

Patients: Arab Bedouin patients with known CD or UC were included in the present study. Fifty patients of 68 Bedouin Arab IBD patients in southern Israel were available for collection of the blood sample.

Demographic and clinical data for all included participants were reviewed carefully. The Data on demographics, the extent of disease, medical therapy, surgery, disease classification, complications, smoking and family history of IBD were obtained via questionnaire and review of patient's records.

For $\mathrm{CD}$ and UC, the Montreal classification was used as previous described [19].

The study was carried out in accordance with the principles of the Helsinki Declaration. The study protocol was approved by the Institutional Ethics Committee, Helsinki committee at the Soroka University Medical Center, the approval number is $0036-14$.

\section{ASCA}

Patient serum samples were analyzed by a standardized enzyme-linked immunosorbent assay (ELISA) using antibodies ASCA IgG and also obtained by using INOVA QUANTA LiteTM ASCA kit [20].
The reactivity for each sample were calculated by dividing the average absorbance of the sample by the average absorbance of the ASCA IgG ELISA Low Positive and is multiplied by the number of units assigned to the ASCA IgG ELISA Low Positive.

Results of $0-20$ was considered as negative, and 20.124.9 as equivocal and $\geq 25$ as positive [20].

The sensitivity of the assay was reported previous as $74.4 \%$ for CD and $14.2 \%$ for UC [20].

\section{ANCA}

ANCA testing was performed using Immunofluorescence analysis of pANCA, cANCA and atypic ANCA by a kit from Inova Diagnostic inc, San Diego, CA USA. The positive results were therefor analyzed for antiMyeloperoxidase (MPO) antibodies and anti proteinase3 (PR3) [21].

Negative Reaction of ANCA was considered negative if specific nuclear and cytoplasmic staining is equal to or less system Negative Control. Positive sample was considered if specific nuclear and/or cytoplasmic staining was observed to be greater than the negative control [21].

\section{Statistical analysis}

The results are presented as the mean $( \pm \mathrm{SD})$ for continuous variables and the percentage of total patients for categorical data. The statistical analysis was carried out using SPSS IBM, version 21. For the categorical variables, proportions were compared using a t-test or $\chi^{2}$, as appropriate.

Significant differences in ASCA titers for different CD phenotypes were assessed using the Mann-Whitney $U$ test. A $p$-value less than or equal to 0.05 was considered statistically significant.

\section{Results}

A total of 50 IBD Bedouin patients, $25 \mathrm{CD}$ and $25 \mathrm{UC}$ were enrolled in our study, which accounts for $73 \%$ of the entire cohort of Bedouin Arab IBD patients in southern Israel.

\section{ASCA and ANCA frequency}

The ASCA and ANCA frequency in CD and UC patient are summarized in Table 1. ASCA is more common among $\mathrm{CD}$ patients, with $52 \%$ of the $\mathrm{CD}$ patients compared with $12 \%$ in UC patients $(p=0.002)$. Among the 25 included UC patients, the most common type of ANCA was atypical ANCA, 36\% (9/25).

\section{ASCA and CD}

No significantly differences were found regarding age and age at diagnosis among ASCA positive and negative $\mathrm{CD}$ patients. None of the other clinical parameters including localization of the disease, surgery or treatment 
Table 1 The frequency of ASCA and ANCA among CD and UC patients

\begin{tabular}{lll}
\hline & $\begin{array}{l}\mathrm{CD} n=25 \\
(\%)\end{array}$ & $\begin{array}{l}\cup C n=25 \\
(\%)\end{array}$ \\
\hline $\begin{array}{l}\text { ASCA-positive } \\
\text { ANCA-positive }\end{array}$ & $3(12)$ \\
PANCA & $152)$ & \\
CANCA & $2(8)$ & $6(24)$ \\
atypical ANCA & $2(8)$ & $4(16)$ \\
\hline
\end{tabular}

with Anti-tumor necrosis factor (anti-TNF) was significantly different among the ASCA positive patients compared with the ASCA negative CD patients. The clinical characteristics of the CD patients are summarized in Table 2.

\section{ANCA and UC}

For the UC group, all three types of ANCA were found; positive c-ANCA was detected in 16\% (4/25), p-ANCA in $24 \%(6 / 25)$ and atypical ANCA in $36 \%(9 / 25)$.

Of the patients with UC with negative ANCA or cANCA 90\% (9/10) were male, while among patients with positive atypical ANCA or positive pANCA only $20 \%(3 / 15)$ were male $(p=0.002)$.

In the comparison between patients who tested positive for ANCA with those who tested negative for ANCA, we found out that the patients with positive ANCA were younger, 40.2 \pm 11.9 years compared with $57.3 \pm 21.2$ years $(p=0.03)$ and the age at diagnosis was $29.2 \pm 11.8$ years compared $43.5 \pm 15.3$ years $(p=0.05)$. There was no relationship regarding other clinical parameters that was found. The clinical characteristics of the UC patients are summarized in Table 3.

Table 2 Clinical Characteristics of ASCA-positive and ASCAnegative CD disease cohort

\begin{tabular}{llll}
\hline Characteristic & $\begin{array}{l}\text { ASCA positive } \\
n=13(52 \%)\end{array}$ & $\begin{array}{l}\text { ASCA negative } \\
n=12(48 \%)\end{array}$ & $p$-value \\
\hline Age & $35.0 \pm 9.1$ & $38.1 \pm 11.6$ & 0.65 \\
Age at diagnosis & $26.2 \pm 6.9$ & $27.6 \pm 9.8$ & 0.89 \\
Gender-male & $8(61.5)$ & $7(58.3)$ & 0.87 \\
Appendectomy & $3(25)$ & $2(18.2)$ & 0.69 \\
Family history of IBD & $2(15.4)$ & $3(25)$ & 0.54 \\
Smoking & $5(38.5)$ & $3(25)$ & 0.47 \\
Anti TNF treatment & $6(50)$ & $4(33.3)$ & 0.43 \\
Passed surgery & $4(30.8)$ & $6(50)$ & 0.32 \\
Disease localization & & & 0.61 \\
Ileal & $5(38)$ & $4(33.3)$ & 0.61 \\
L2 & $0(0)$ & $2(16.7)$ & 0.61 \\
L3 & $6(46)$ & $6(50)$ & 0.61 \\
L4 & $2(15.4)$ & $0(0)$ & \\
\hline & & & \\
\hline
\end{tabular}

Table 3 Clinical Characteristics of ANCA-positive UC patients

\begin{tabular}{|c|c|c|c|c|}
\hline Characteristic & $\begin{array}{l}\text { CANCA } \\
n=4(16 \%)\end{array}$ & $\begin{array}{l}\text { pANCA } \\
n=6(24 \%)\end{array}$ & $\begin{array}{l}\text { atypicANCA } \\
n=9(36 \%)\end{array}$ & $\overline{p \text {-value }}$ \\
\hline Age & $44.0 \pm 17.6$ & $35.5 \pm 14.2$ & $41.6 \pm 7.4$ & 0.52 \\
\hline Age at diagnosis & $23.0 \pm 13.6$ & $26.8 \pm 14.1$ & $33.5 \pm 8.6$ & 0.29 \\
\hline Gender-male & $4(100)$ & $1(16.7)$ & $2(22.2)$ & 0.01 \\
\hline Appendectomy & $0(0)$ & $0(0)$ & $0(0)$ & - \\
\hline Family history & $0(0)$ & $1(16.7)$ & $0(0)$ & 0.32 \\
\hline Smoking & $1(25.0)$ & $1(16.7)$ & $0(0)$ & 0.33 \\
\hline Passed surgery & $1(25.0)$ & $0(0)$ & $1(11.1)$ & 0.49 \\
\hline \multicolumn{5}{|l|}{ Localization } \\
\hline Proctitis & $0(0)$ & $1(16.7)$ & $2(22.2)$ & 0.38 \\
\hline left Colitis & $1(25.0)$ & $4(66.7)$ & $3(33.3)$ & 0.38 \\
\hline Pancolitis & $3(75.0)$ & $1(16.7)$ & $4(44.4)$ & 0.38 \\
\hline
\end{tabular}

\section{Discussion}

IBD prevalence in Bedouin Arab population is increasing [2]. ASCA and ANCA are very well-studied markers in $\mathrm{CD}$ and $\mathrm{UC}$ as diagnostic markers or as a predictor for disease phenotype. No data were reported regarding these serological markers in the Arab Bedouin population.

We investigated the frequency of these markers and its association with the disease phenotype. This is the first report of IBD serologic markers Arab Bedouin IBD patients in southern Israel.

ASCA are known to be predominantly associated with CD and ANCA with UC.

In our study we found the frequency of $52 \%$ of ASCA in $\mathrm{CD}$ patients. Among the UC patients, we also found a frequency of $36 \%$ of atypical ANCA, whereas the frequency of pANCA was $24 \%$ of the UC cohort.

The frequency range of ASCA and ANCA are so wide and different in literature and in different population. The frequency of ASCA in CD patients and ANCA in UC patients may reach $80 \%$ in some reports [9-14].

In this study, we found a high frequency of ASCA and pANCA. However, in our cohort the most frequent ANCA type was the atypical ANCA and not the pANCA.

There are only few reports in the Arab world regarding serological markers in IBD among the Arab population; a study of IBD in children from Saudi Arabia found a prevalence of $35 \%$ of ASCA in CD patients and $28 \%$ of pANCA in UC [22].

Bread and beer are important sources of Saccharomyces cerevisiae yeast, only scant paper published regarding the relationship between the diet and ASCA frequency. However, no support of relationship between bread consumption and ASCA frequency was found in the scientific literature [23].

An important part of the nutrition among the BA is bread, which is included in the daily nutrition [24], and a low percentage of alcohol, we found a relative high 
frequency of ASCA among CD patients, the possible relationship between high consumption of bread and frequency of ASCA among this population is still to investigate in the future.

On the basis of the results of the present study, no relationship was found between the ASCA positivity and the disease phenotype characteristics in the $\mathrm{CD}$ patient's cohort.

Also, in previous literature there are a large number of reports regarding the relationship between ASCA and CD phenotype; however, the results are controversial and vary among the previously reported studies, some of them found an association between ASCA positivity and early disease onset, longer disease duration, ileal involvement, complicated disease and IBD-related surgery [13-18]. Other several independent studies found no association between these parameters and ASCA positivity [25], it is therefore, important to mention is that these studies were heterogeneous in term of design, inclusion criteria, number of patients and other parameters of methodology [25]. In our work, the patients with positive ASCA were younger; however, it's still none statistically significant. In our cohort we didn't find a relationship between ASCA positivity and gender, family history of IBD, smoking, disease location, anti-TNF treatment or surgery.

While it is possible that there is no relationship between ASCA and disease phenotype in our population, from another view the no relationship perhaps could be linked to the small number of IBD patients in this specific population. It has also been suggested that to investigate this issue in this specific population in the future, when the number of IBD patients is larger, which could make the relationship clearer.

According to our results, the most common type of ANCA in Arab Bedouin IBD patients is the atypical ANCA, which was common by $36 \%$. Several previous reports indicated a high frequency of atypical ANCA in other populations $[26,27]$. In the present research the frequency of pANCA was $24 \%$. This, therefore, translate into $60 \%$ of our UC cohort tested positive for pANCA or Atypical ANCA. In comparing our results with those in previous literature particularly Arab children in Saudi children, which showed a $28 \%$ frequency of pANCA [22], the frequency of pANCA and atypical ANCA is high in our cohort. To the best of our knowledge there are no other reports from Arab population in the Arab World.

In contrast to the more accurate results seen in our UC cohort, we have demonstrated that male patients are more likely to have negative ANCA or cANCA rather than atypical or pANCA. Of the ten patients who had negative ANCA or positive cANCA, nine of them were male (90\%), however only 3 of 15 patients (20\%) with positive pANCA or atypical ANCA were male $(p=0.002)$.
Atypical ANCA testing might be a helpful test for differentiating UC from CD in this specific population. Previous study suggested that atypical p-ANCA is a useful parameter to differentiate UC from CD [26].

In one hand, we have reported before, that the IBD among Bedouin Arab is increasing [2], which is attributed to change of lifestyle, including urbanization and modernization with a western lifestyle, particularly change of hygiene and nutrition. In the other hand we found a high prevalence of ASCA and ANCA positivity in the specific population. The Bedouin Arab in southern Israel are a very young society, $60 \%$ of the population are younger than age 19 [1]. The compensation of these factors let us expecting continuation increase of IBD incidence and prevalence in the future. The importance of this study might be the detecting a high frequency of serologic markers and its implication as diagnostic markers or as marker, which could differentiate between CD and UC.

The present study is limited by the small number of patients, the use of only the ASCA IgG type and the lack of control group.

\section{Conclusion}

We found a high prevalence of ASCA in CD patients among Arab Bedouin IBD patients without relationship to the disease phenotype. A high frequency of atypical ANCA and pANCA were found among the UC patients with a prediction for younger age at diagnosis.

\section{Abbreviations}

ANCA: Anti-neutrophil cytoplasmic antibodies; ASCA: Anti-Saccharomyces cerevisiae antibodies; CD: Crohn's disease; IBD: Inflammatory bowel disease; UC: Ulcerative colitis

\section{Acknowledgements \\ Not applicable \\ Funding \\ The study received no funding.}

Availability of data and materials

The datasets used and/or analyzed during the current study are available from the corresponding author on reasonable request.

\section{Authors' contributions}

AFN, FA designed the study. BW and AFN collected the data. EO, EJ, MA and $\mathrm{SA}$ were involved in the analysis and interpretation of the data. SBI were responsible for the laboratory work. ATM made the statistical analysis. AFN, FA, EO were major contributors to writing the manuscript. All authors read and approved the final manuscript.

\section{Ethics approval and consent to participate}

The study protocol was approved by the Institutional Ethics Committee, Helsinki committee at the Soroka University Medical Center, the approval number is 0036-14. A written informed consent was obtained from all participants.

Consent for publication Not applicable 


\section{Competing interests}

The authors declare that they have no competing interests.

\section{Publisher's Note}

Springer Nature remains neutral with regard to jurisdictional claims in published maps and institutional affiliations.

\section{Author details}

${ }^{1}$ The Institute of Gastroenterology and Hepatology, Soroka University Medical Center and the Faculty of Health Sciences, Ben-Gurion University of the Negev, P.O. Box 151, 84101 Beer-Sheva, Israel. ${ }^{2}$ Internal Medicine ward E, Soroka University Medical Center, Beer-Sheva, Israel. ${ }^{3}$ Department of Clinical Biochemistry and Pharmacology, Ben-Gurion University of the Negev, Beer-Sheva, Israel. ${ }^{4}$ Nursing Research Unit, Soroka University Medical Center Beer-Sheva, Israel.

\section{Received: 7 January 2018 Accepted: 14 October 2018}

\section{Published online: 20 October 2018}

\section{References}

1. Central Statistics Bureau. Available in csb.co.il. Accessed 15 Oct 2016

2. Abu Freha N, Schwartz D, Elkrinawi J, Ben Yakov G, Abu Tailakh M Munteanu D, et al. Inflammatory bowel disease among Bedouin Arab in southern Israel. Urbanization and increasing prevalence rates. Eur J Gastroenterol Hepatol. 2015;27(3):230-4.

3. Main J, McKenzie $H$, Yeaman GR, Kerr MA, Robson D, Pennington CR, et al. Antibody to Saccharomyces cerevisiae (bakers' yeast) in Crohn's disease. BMJ. 1998;297:1105-6.

4. Savige J, Dimech W, Fritzler M, Goeken J, Hagen EC, Jennette JC, et al. Addendum to the international consensus statement on testing and reporting of Antineutrophil cytoplasmic antibodies. Quality control guidelines, comments, and recommendation for testing in other autoimmune disease. Am J Clin Pathol. 2003;120:312-8.

5. Kallenberg CGM, Heeringa P, Stegeman CA. Mechanisms of disease: pathogenesis and treatment of ANCA-associated vasculitides. Nat Clin Pract Rheumatol. 2006;2:661-70.

6. Editorial. Rational use of ANCA in diagnosis of vasculitis. Rheumatology. 2002:41:481-3

7. Papp M, Altorjay I, Lakos G, Tumpek J, Sipka S, Dinya T, et al. Evaluation of the combined application of ethanol-fixed and formaldeyde-fixed neutrophil substrates for identifying atypical perinuclear antineutrophil cytoplasmic antibodies in inflammatory bowel disease. Clin Vaccine Immunol. 2009;16(4):464-70.

8. Lochman I, Kral V, Lochmanova A, Lupač J, Cebecauer L. ANCA in the diagnosis of neutrophil-mediated inflammation. Autoimmun Rev. 2011;10: 295-8.

9. Quinton JF, Sendid B, Reumaux D, Duthilleul P, Cortot A, Grandbastien B, et al. Anti-Saccharomuces cerevisiae mannan antibodies combined with antineutrophil cytoplasmic autoantibodies in inflammatory bowel disease: prevalence and diagnostic role. Gut. 1998;42:788-91.

10. Sandborn WJ, Loftus EV Jr, Colombel JF, Fleming KA, Seibold F, Homburger $H A$, et al. Evaluation of serologic disease marker in a population-based cohort of patients with ulcerative colitis and Crohn's disease. Inflamm Bowel Dis. 2001;7:192-201.

11. Peeters $M$, Joossens $S$, Vermeirs $S$, Vlietinck $R$, Bossuyt $X$, Rutgeerts $P$. Diagnostic value of anti-Saccharomyces cerevisiae and antineutrophil cytoplasmic autoantibodies in inflammatory bowel disese. Am J Gastroentol. 2001;96:730-4

12. Mokrowiecka A, Daniel P, Slomka M, Majak P, Malecka-Panas E. Clinical utility of serological markers in inflammatory bowel disease. Hepatogastreoenterology. 2009:56(89):162-6.

13. Israeli E, Grotto I, Gilburd B, Balicer RD, Goldin E, Wiik A, et al. AntiSaccharamyces cerevisiae antibodies as predictors of inflammatory bowel disease. Gut. 2005;54:1232-6.

14. Papp M, Norman GL, Altorjay I, Lakatos PL. Utility of serological markers in inflammatory bowel diseases: gadget or magic? World J Gastroenterol. 2007;13(14):2028-36

15. Vasiliauskas EA, Kam LY, Karp LC, Gaiennie J, Yang H, Targan SR. Marker antibody expression stratifies Crohn's disease into immunologically homogenous subgroups with distinct clinical characteristics. Gut. 2000; 47:487-96.
16. Sastegni R, Daperno M, Ercole E, Rigazio C, Bresso F, Masoero G, et al. Detection of anti-Saccharomyces cerevisiae antibodies in Crohn's disease: is it a reliable diagnostic and prognostic marker. Dig Liver Dis. 2001;33:755-61.

17. Forcione DG, Rosen MJ, Kisiel JB, Sands BE. Anti-Saccharomyces cerevisiae antibody (ASCA) positivity is associated with increased risk for early surgery in Crohn's disease. Gut. 2004;53:1117-22.

18. Zhang Z, Li C, Zhao X, LV C, He Q, Lei S, et al. Anti-Saccharomyces cerevisiae antibodies associate with phenotypes and higher risk for surgery in Crohn's disease: a meta-analysis. Dig Dis Sci. 2012;57:2944-54.

19. Silverberg MS, Satsangi J, Ahmad T, Arnott ID, Bernstein CN, Brant SR, et al. Toward an integrated clinical, molecular and serological classification of inflammatory bowel disease: report of a Working Party of the 2005 Montreal World Congress of Gastroenterology. Can J Gastroenterol. 2005;19(A):5A-36A.

20. http://www.inovadx.com/PDF/clsi/708865_clsi_EN.pdf. Accessed 21 Apr 2018

21. http://www.inovadx.com/PDF/di/708290 EN.pdf. Accessed 21 Apr 2018.

22. Saadah O, Al-Mughales J. Serological markers of inflammatory bowel disease in children from the Western region of Saudi Arabia. Arab J Gastroenterol. 2013;14:78-82.

23. Kvehaugen AS, Aasbrenn M, Farup PG. Anti-Saccharomyces cerevisiae antibodies (ASCA) are associated with body fat mass and systemic inflammation, but not with dietary yeast consumption: a cross-sectional study. BMC Obes. 2017:4:28.

24. Abu-Saad K, Shahar DR, Vardi H, Fraser D. Importance of ethnic foods as predictors of and contributors to nutrient intake levels in a minority population. Eur J Clin Nutr. 2010;64(3):S88-94.

25. Bonneau J, Dumestre-Perard C, Rinaudo-Gaujous M, Genin C, Sparrow M, Roblin $X$, et al. Systemic review: new serological markers (anti-glycan, antiGP2, anti-GM-CSF ab) in the prediction of IBD patient's outcomes. Autoimmun Rev. 2015;14:231-45.

26. Mokhtarifar A, Ganji A, Sadrneshin M, Bahari A, Esmaeilzadeh A Ghafarzadegan K, et al. Diagnostic value of ASCA ant atypical p-ANCA in differential diagnosis of inflammatory bowel disease. Middle East J Dig Dis. 2013:5:93-7.

27. Bouzid D, Haddouk S, Amouri A, Ben Hadj Hmida Y, Tahri N, Masmoudi H. Contribution of immunofluorescence to identification and characterization of antineutrophil cytoplasmic antibodies in inflammatory bowel disease. Indian J Gastroenterol. 2011;30:229-32.

Ready to submit your research? Choose BMC and benefit from:

- fast, convenient online submission

- thorough peer review by experienced researchers in your field

- rapid publication on acceptance

- support for research data, including large and complex data types

- gold Open Access which fosters wider collaboration and increased citations

- maximum visibility for your research: over $100 \mathrm{M}$ website views per year

At $\mathrm{BMC}$, research is always in progress.

Learn more biomedcentral.com/submission 